\title{
Nasopharyngeal Metastasis Originating From Beta-HCG Being Secreting From Transitional Cell Carcinoma of the Renal Pelvis: A Case Report
}

\author{
Arun Vasudevan ${ }^{1}$, Lakshmi Haridas $\mathrm{K}^{1, *}$, Venugopal Muraleedharan ${ }^{2}$ \\ ${ }^{1}$ Department of Medical Oncology, Regional Cancer Center, Trivandrum, \\ Kerala, India \\ ${ }^{2}$ Department of Radiodiagnosis, Regional Cancer Center, Trivandrum, Ker- \\ ala, India
}

*Corresponding author: Lakshmi Haridas K, Department of Medical Oncology, Regional Cancer Center, Trivandrum 695011, India. Tel: +919447206106; E-mail:lakshmiharidasrcc@gmail.com

DOI: $10.30699 /$ mci.4.4.435

Submitted: 5 July 2020

Revised: 19 August 2020

Accepted: 21 September 2020

e-Published: 1 October 2020

Keywords:

Carcinoma, Transitional Cell

Kidney Pelvis

Chorionic Gonadotropin
Introduction: Transitional cell carcinomas (TCC) of the upper urinary tract constitute only $7 \%$ of urothelial carcinomas. The majority of upper urinary tract TCC arises from the renal pelvis, followed by the ureter. The majority of the patients are presented with microscopic or macroscopic haematuria, flank pain, or symptoms due to metastasis; however, it can be asymptomatic in $10-15 \%$.

Case presentation: Here, we report the case of a 58-year-old male who was referred with headache and cranial nerve palsy, later diagnosed with beta-human chorionic gonadotropin (beta-HCG) that was secreted from TCC of the renal pelvis with nasopharyngeal, lung, and brain metastasis.

Conclusion: Nasopharyngeal metastasis from TCC of the renal pelvis is a rare entity and this case report adds to the present literature.

\section{INTRODUCTION}

Transitional cell carcinomas (TCCs) of the upper urinary tract constitute $7 \%$ of urothelial carcinomas. They are significantly less common than renal cell carcinomas. The majority of upper urinary tract TCCs arise from the renal pelvis, followed by the ureter [1]. It is mostly seen in male patients above 50 years of age. While the disease is asymptomatic in $10-15 \%$ of patients, it is symptomatic in the rest of cases with microscopic or macroscopic haematuria, flank pain, or symptoms caused by metastasis [1]. The most common sites of metastasis include the liver, lungs, and bone [1]. Unusual metastasis to skin, clitoris, duodenum, and larynx has been reported in the literature [2-4]. However, metastasis to nasopharynx from urothelial carcinoma of the renal pelvis has not yet been reported. Here, we report the case of a 58-year-old male who presented with headache and cranial nerve palsy, later diagnosed with beta-human chorionic gonadotropin (betaHCG) being secreted from TCC of the renal pelvis with nasopharyngeal, lung, and brain metastasis.

\section{CASE PRESENTATION}

A 58-year-old male, with no major comorbidities 
who was a current smoker, was evaluated for a three-month history of headache, diplopia, and left nasal block. No other organ-related symptoms/ history of recurrent urinary tract infection was elicited, although he reported occasional hemoptysis during the past fortnight. He reported no addictions, drug abuse, or taking chronic medications. At the initial presentation, his Eastern Co-operative Oncology Group (ECOG) performance status was 3 with severe headache and dyspnea on exertion. Physical examination showed left ophthalmic palsy (lateral rectus) and a boggy swelling involving the left epigastric, lumbar, and umbilical area. External genitalia was normal. Contrast-Enhanced Computed Tomography (CECT) of the abdomen showed grossly enlarged left kidney with severe parenchymal thinning and non-dependent enhancing nodules in the renal pelvis (Figure 1A and 1B). The left pelvicalyceal system was hugely dilated and ballooned out to cross the midline towards the right side. The left ureteropelvic junction (UPJ) was untraceable; while the left ureter was normally traced from L5-S1 level up to the left vesicoureteric junction. The right kidney was unremarkable. Chest CT showed multiple bilateral nodules with peritumoral hemorrhage and cavitary changes suggestive of metastasis (Figure 1C). Magnetic Resonance Imaging (MRI) of the brain showed an irregular ill-defined enhancing lesion involving the left clivus and skull base, suspicious of metastasis (Figure 1D). His hemogram, coagulation profile, renal/lung function tests, c-antineutrophil cytoplasmic antibodies, Quantiferon-TB Gold were within the normal limits. Cystoscopy was normal. Ureteroscopy was attempted, but the procedure could not be completed due to respiratory compromise on-table. His serum beta-HCG was elevated, the highest being $2531 \mathrm{mIU} / \mathrm{mL}(0.5-6.5)$. Urine betaHCG was also positive. Ultrasonography guided Fine Needle Aspiration Cytology of the renal mass was suggestive of carcinoma (CK 7+ve; CK20-ve). Nasopharyngeal endoscopy showed an ulcerated lesion filling the entire left side of the nasopharynx. Biopsy from the nasopharyngeal lesion confirmed high grade poorly differentiated carcinoma (Figure $2 \mathrm{~A}$ and $2 \mathrm{~B}$ ). On immunohistochemistry (IHC), the tumor cells were immunonegative for CK-20, TTF-1, SALL4, OCT4, PAX8, Uroplakin, \& AFP and immunoreactive for CK-7, p63, GATA-3,
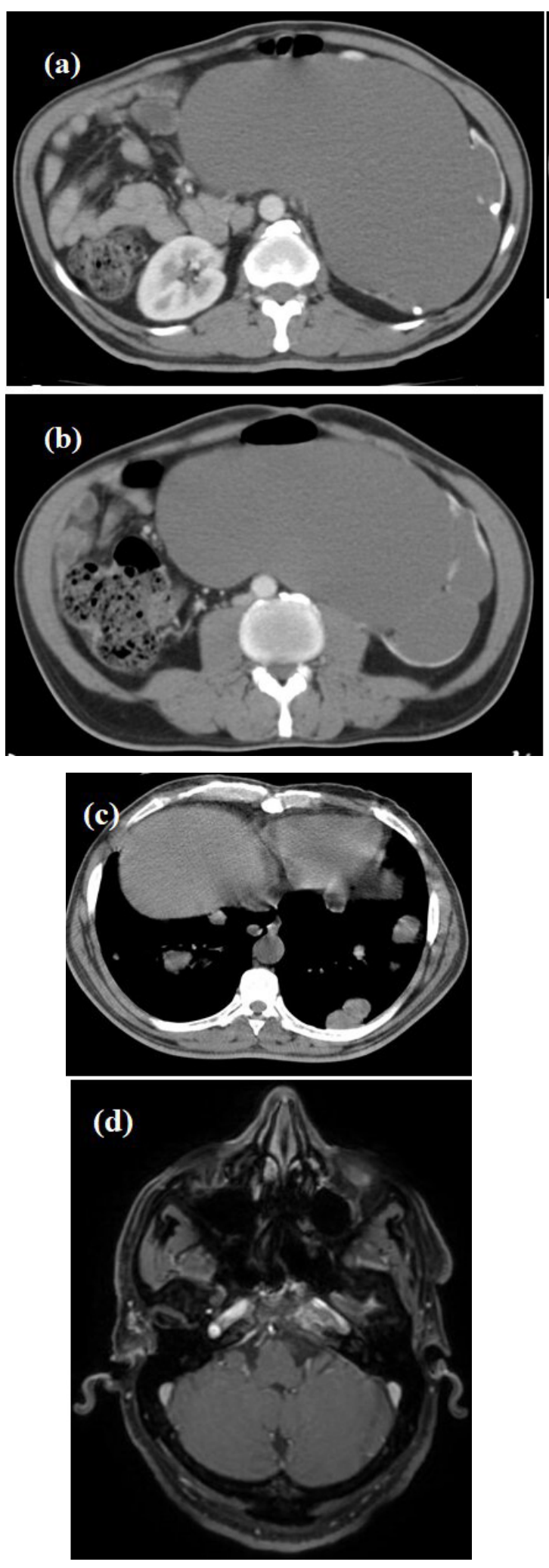

Figure 1: Radiological Images

(A \& B) Computed tomography of the abdomen grossly enlarged left kidney with severe parenchymal thinning; (C) Chest computed tomography showing bilateral lung metastases; (D) Contrast magnetic resonance imaging of the brain (T1) Showing poorly defined enhancement in left clivus, suggestive of metastasis 


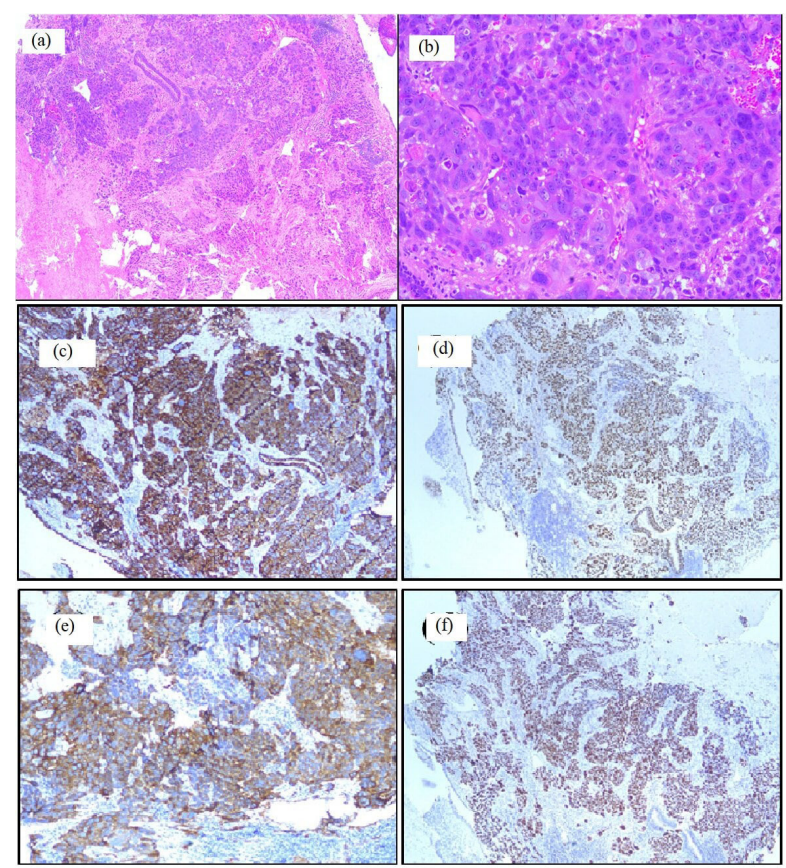

Figure 2: Histopathology Images

(A \& B) H\&E stained nasopharyngeal biopsy $(5 \times)$, and $(20 \times)$ respectively. Tumor cells with enlarged, pleomorphic nucleus, nucleoli, and eosinophilic cytoplasm are seen as nests amidst fibrotic stroma, suggesting high grade poorly differentiated carcinoma; (C \& D \& E \& F) Immunohistochemistry (IHC) nasopharyngeal biopsy showing CK-7, p63, Glypican -3, GATA3 positivity respectively

Glypican, \& beta-HCG (occasional)(Figure 2C2F). In-situ hybridization for Epstein-Barr virus - ribonucleic acid was negative. Histopathologic features suggested metastatic high-grade urothelial carcinoma. After a multidisciplinary tumor board discussion, a diagnosis of TCC in the left renal pelvis with metastasis to nasopharynx, lung, and brain was made. He underwent palliative radiation to the nasopharynx and base of the skull - 20Gy in five fractions and was given two cycles of cisplatinbased palliative chemotherapy, after which he was lost to follow up.

\section{DISCUSSION}

TCC of the renal pelvis is uncommon compared to renal cell carcinoma. The former is predominantly seen in men aged $>50$ years. Other risk factors are smoking, phenacetin over usage, human papillomavirus infection, Balkan nephropathy, Blackfoot disease, etc [1]. Our patient was a male smoker in his sixth decade of life. Patients often present with haematuria (microscopic/macroscopic), loco-regional symptoms, or symptoms caused by distant metastasis. The primary lesion can be asymptomatic in $10-15 \%$ of patients, like in the present case, where the patient was asymptomatic for the primary lesion and was presented with signs of metastasis, i.e, headache, cranial nerve palsy, and hemoptysis. Abdominal CT often shows soft tissue density lesion (8-10HU) centered in the renal pelvis which enhances less compared to renal parenchyma with either a small filling defect or as large mass distorting the renal sinus. In this patient, the involved pelvicalyceal system was ballooned out along with extreme thinning of the renal parenchyma and non-traceable pelviureteric junction. Clivus erosion and hemorrhagic lung metastasis showed a huge metastatic tumor load in this case. During the workup, we found that the patient's serum beta-HCG was high which was demonstrable in the urine as well. Upon literature review, it was concluded that $12 \%$ of urothelial carcinomas can have syncytiotrophoblastic differentiation, either due to metaplastic differentiation or pluripotency of the native urothelial lining, thereby producing beta-HCG which can act in paracrine/autocrine fashion [5]. There are several reported cases of urothelial carcinomas of the bladder with beta-HCG production [6]. Generally, beta-HCG secreting urothelial carcinomas are high grade, with higher metastatic potential and poor response to the treatment, thereby carrying a dismal prognosis [5]. The pathological diagnosis of such an entity is made after demonstrating TCC components along with the syncytiotrophoblasts [7]. Similarly, the biopsy of the present patient showed TCC with beta-HCG positivity on IHC, thereby correlating with the patient's clinical beta-HCG reports and metastatic burden. To the best of our knowledge, this is the first case report of TCC of the renal pelvis with betaHCG production.

Nasopharyngeal metastasis from renal cell carcinoma is well reported in the literature [8]. Similarly, TCC of the bladder has been reported to metastasize to unusual sites including testis, mandible, shoulder girdle, etc. [9-11]. Our case adds to the present literature as the first to present with nasopharyngeal metastasis from a beta-HCG secreting primary TCC of the renal pelvis. Possible mechanisms of such unusual metastasis from kidney cancer may be due to the rich inherent pressurized vascular nature of the organ and its access to the 
hematogenous and lymphatic system. In the present patient, possibly both the routes were involved in producing nasopharyngeal, lung, and brain metastasis. Endoscopic biopsy is warranted in such a case to rule out a primary nasopharyngeal carcinoma or benign conditions like Wegner granulomatosis and tuberculosis. The prognosis for metastatic TCC is extremely poor with a probable 1 -year survival of $35 \%$ [12]. The best intent of treatment in metastatic disease is palliation. We have treated our patient by extrapolating the guidelines of metastatic TCC of the bladder, with systemic chemotherapy. $\mathrm{He}$ also received palliative radiotherapy to the nasopharynx and base of the skull. The standard treatment options, as per National Comprehensive Cancer Network (NCCN) guidelines, in cisplatineligible patients, are gemcitabine-cisplatin/ carboplatin as well as combination chemotherapy with methotrexate, vinblastine, doxorubicin, and cyclophosphamide (MVAC). In cisplatin-ineligible cases, immunotherapy choices have recently come into practice. It would be worthwhile to note that recent molecular studies revealed differences between upper urinary tract urothelial cancer and bladder cancer in terms of molecular/clinical subtypes and possibly contribute to differential treatment outcome [13]. More insight is needed in this area for future refinements in treatment.

\section{ACKNOWLEDGEMENTS}

None declared.

\section{CONFLICTS OF INTEREST}

The authors declared no conflict of interest.

\section{ETHICS APPROVAL}

Informed consent obtained.

\section{REFERENCES}

1. Kirkali Z, Tuzel E. Transitional cell carcinoma of the ureter and renal pelvis. Crit Rev Oncol Hematol. 2003;47(2):155-69.

2. Singh P, Kolte S, Singh GK. Cytological diagnosis of a rare case of cutaneous metastasis from transitional cell carcinoma, renal pelvis. J Egypt Natl Canc Inst. 2017;29(4):197-
200. DOI: 10.1016/j.jnci.2017.11.001 PMID: 29233453.

3. Sakin YS, Kekilli M, Celebi G, Ozturk K, Uygun A, Bagci S. A Rare Case of Upper Gastrointestinal Bleeding: Duodenal Metastasis of Transitional Cell Carcinoma Originating from Renal Pelvis. J Gastrointest Cancer. 2015;46(3):3256. DOI: 10.1007/s12029-015-9703-6 PMID: 25792484.

4. Assi T, El Rassy E, Haddad A, Kattan J. Solitary Laryngeal Metastasis from Transitional Cell Carcinoma of the Kidney: Clinical Case and Review of the Literature. Case Rep Otolaryngol. 2015;2015:595283. DOI: 10.1155/2015/595283 PMID: 26236525.

5. Yacur M, Marks EI, Sulseki J, Drabick JJ, Crist H, Joshi M. Expression of Beta-Human Chorionic Gonadotropin (Beta-HCG) in Transitional Cell Carcinoma. Ann Carcinog. 2017;2(1):1012.

6. Venyo AK, Herring D, Greenwood H, Maloney DJ. The expression of beta human chorionic gonadotrophin (beta-HCG) in human urothelial carcinoma. Pan Afr Med J. 2010;7:20. PMID: 21918707.

7. Zhai QJ, Black J, Ayala AG, Ro JY. Histologic variants of infiltrating urothelial carcinoma. Arch Pathol Lab Med. 2007;131(8):1244-56. DOI: 10.1043/1543-2165(2007)131[1244:HVOIUC]2.0.CO;2 PMID: 17683187.

8. Atar Y, Topaloglu I, Ozcan D. Metastatic renal cell carcinoma in the nasopharynx. Indian J Pathol Microbiol. 2013;56(1):40-2. DOI: 10.4103/0377-4929.116147 PMID: 23924557.

9. Kozak GN, Field NC. Metastatic transitional cell carcinoma of the bladder to the testis: a case report. Case Rep Urol. 2012;2012:486245. DOI: 10.1155/2012/486245 PMID: 23133785 .

10. Siriwardena BSMS, Tilakaratne WM, Rajapaksa RMSK Metastatic transitional cell carcinoma of the urinary bladder to the mandible. Oral Oncol Extra. 2005;41(2):22-4 DOI: 10.1016/j.ooe.2004.10.001.

11. Weizer AZ, Shariat SF, Haddad JL, Escudier S, Lerner SP. Metastatic transitional cell carcinoma of the urinary bladder to the shoulder girdle. Rev Urol. 2002;4(2):97-9. PMID: 16985663.

12. Halim A, Abotouk N. Methotrexate-paclitaxel-epirubicin-carboplatin as second-line chemotherapy in patients with metastatic transitional cell carcinoma of the bladder pretreated with cisplatin-gemcitabine: a phase II study. Asia Pac J Clin Oncol. 2013;9(1):60-5. DOI: 10.1111/j.17437563.2012.01554.x PMID: 22897883.

13. Bianconi M, Cimadamore A, Faloppi L, Scartozzi M, Santoni M, Lopez-Beltran A, et al. Contemporary best practice in the management of urothelial carcinomas of the renal pelvis and ureter. Ther Adv Urol. 2019;11:1756287218815372. DOI: $10.1177 / 1756287218815372$ PMID: 30671136. 